\title{
La curva medioambiental de Kuznets y el crecimiento económico sostenible en Colombia
}

\author{
Camilo Fabiam Gómez Segura* \\ Óscar Hernán Cerquera Losada ** \\ Edwin Fernando Acero Cebay ***
}

Fecha de recepción: 7 de julio de 2020

Fecha de aprobación: 3 de diciembre de 2020

Resumen: El presente trabajo busca comprobar empíricamente la hipótesis de la curva medioambiental de Kuznets (CMK) en las emisiones de dióxido de carbono $\left(\mathrm{CO}_{2}\right)$ y óxido nitroso $\left(\mathrm{N}_{2} \mathrm{O}\right)$ durante el periodo 1990-2012 para 217 economías, utilizando la base de datos del Banco Mundial. Se realizó un modelo de datos panel bajo tres alternativas (efectos fijos, efectos aleatorios y primeras diferencias). Los resultados obtenidos muestran que existe una relación de $\mathrm{U}$ invertida entre la contaminación ambiental $\left(\mathrm{CO}_{2}\right.$ y $\left.\mathrm{N}_{2} \mathrm{O}\right)$ y el producto interno bruto (PIB) per cápita como medida de crecimiento económico, siendo el método de efectos fijos el que genera estimaciones consistentes al aplicar la prueba de Hausman. No obstante, la relación entre contaminación y crecimiento económico presenta rendimientos decrecientes muy bajos, lo cual genera elevados puntos de inflexión principalmente en las emisiones de $\mathrm{CO}_{2}$.

Palabras clave: curva medioambiental de Kuznets; contaminación ambiental; crecimiento económico; desarrollo económico; cambio tecnológico; panel de datos.

Clasificación JEL: Q01, Q50, Q51, Q53, Q56.

Cómo citar este artículo:

Gómez, C.; Cerquera, O. \& Acero E. (2021). La curva medioambiental de Kuznets y el crecimiento económico sostenible en Colombia. Apuntes del Cenes, 40(71). Págs. 165 - 188 https://doi.org/10.19053/01203053.v40. n71.2021.11736

* Magíster en Economía. Docente de tiempo completo, Universidad Surcolombiana, Neiva, Colombia. Integrante de grupo de investigación Iguaque; investigador junior del Ministerio de Ciencia, Tecnología e Innovación. camilo. gomez@usco.edu.co iD https://orcid.org/0000-0003-0209-1383

** Magíster en Economía. Docente de tiempo completo, Universidad Surcolombiana, Neiva, Colombia. Integrante de grupo de investigación Iguaque; investigador asociado del Ministerio de Ciencia, Tecnología e Innovación. oscar. cerquera@usco.edu.co iD https://orcid.org/0000-0002-7945-6670

*** Especialista en Estadística. Coordinador operativo, Industria Cacaotera del Huila Tolimax, Neiva, Colombia. aceroedwin@hotmail.com (i) https://orcid.org/0000-0002-7869-233X 


\title{
The Environmental Kuznets Curve and Sustainable Economic Growth in Colombia
}

\begin{abstract}
This paper seeks to empirically test the Environmental Kuznets Curve (EKC) hypothesis in the emissions of carbon dioxide $\left(\mathrm{CO}_{2}\right)$ and nitrous oxide $\left(\mathrm{N}_{2} \mathrm{O}\right)$ during the period 1990-2012 for 217 economies, using the World Bank database. A panel data model was carried out under three alternatives (fixed effects, random effects and first differences). The results obtained show that there is an inverted $\mathrm{U}$ relationship between environmental pollution $\left(\mathrm{CO}_{2}\right.$ and $\left.\mathrm{N}_{2} \mathrm{O}\right)$ and Gross Domestic Product (GDP) per capita as a measure of economic growth, with the fixed effects method generating consistent estimates when applying the Hausman test. However, the relationship between pollution and economic growth presents too low decreasing returns, which generates high turning points, mainly in emissions from $\mathrm{CO}_{2}$.
\end{abstract}

Keywords: Environmental Kuznets Curve; environmental pollution; economic growth; economic development; technological change; data panel. 


\section{INTRODUCCIÓN}

¿Es el continuo crecimiento económico de los países el responsable del deterioro ambiental del planeta? O más aún ¿El incremento del ingreso y la riqueza son las variables que explican parte del deterioro ambiental de los recursos naturales? Las respuestas a estos interrogantes son esenciales para diseñar políticas apropiadas de desarrollo. La forma de producción y la composición de los bienes generan una relación negativa de la demanda ambiental y la actividad económica; sin embrago, la evidencia empírica sugiere que los países desarrollados han obtenido una transformación en su estructura económica. Inicialmente, los cambios tecnológicos, procesos de producción y una legislación favorable a la conservación de recursos naturales han sido lo suficientemente importante para compensar los efectos de la actividad económica en el medio ambiente (Grossman \& Krueger, 1995).

Identificar las causas y los efectos del deterioro ambiental ha generado un gran impacto en la sociedad, ejemplo de ello es el gran número de trabajos sobre esta materia y más aún los acuerdos entre países que buscan mejorar su producción sostenible y por ende el cuidado de los recursos naturales (Conferencia Cambio Climático París, 2015). Dado esto, toma relevancia el supuesto de un crecimiento económico sostenible, es decir, se necesita una transformación en la estructura económica y política ambiental que genere el cambio en los procesos productivos para compensar los efectos adversos hacia el medio ambiente.

En este sentido, una alternativa para estimar los efectos de la actividad económica en el medio ambiente es determinar el impacto del crecimiento económico representado por el ingreso nacional y los indicadores de calidad ambiental. Para ello, se utiliza la CMK, la cual establece que la relación empírica entre el nivel de ingreso per cápita y el deterioro ambiental se representa por una curva con forma de $U$ invertida, que revela que países con bajos niveles de ingresos están correlacionados con un incremento en el deterioro ambiental; por su parte, países con niveles de 
ingresos altos tienen una menor contribución en el deterior del medio ambiente (Grossman \& Krueger, 1995).

La Figura 1 muestra la relación de las emisiones de dióxido de carbono $\left(\mathrm{CO}_{2}\right)$ y óxido nitroso $\left(\mathrm{N}_{2} \mathrm{O}\right)$ con respecto al pro- ducto interno bruto (PIB) per cápita para 217 países y economías registradas en el Banco Mundial en el año 2012 durante el periodo 1990-2012. Como se puede observar, para ambos indicadores de deterioro ambiental un aumento del PIB per cápita está relacionado con una disminución en las emisiones de $\mathrm{CO}_{2}$ y $\mathrm{N}_{2} \mathrm{O}$.
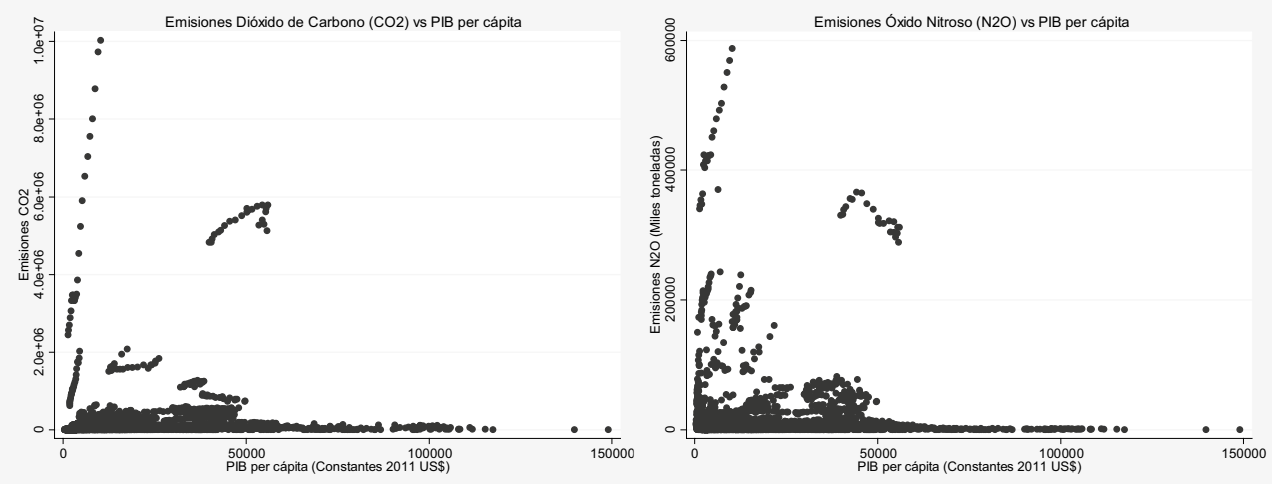

Figura 1. Emisiones de dióxido de carbono $\left(\mathrm{CO}_{2}\right)$ y óxido nitroso $\left(\mathrm{N}_{2} \mathrm{O}\right)$ vs PIB per cápita durante el periodo 1990-2012.

De igual manera, la Figura 2 presenta un diagrama de dispersión entre el crecimiento de las emisiones de $\mathrm{CO}_{2}$ y $\mathrm{N}_{2} \mathrm{O}$ con relación al PIB. Los datos muestran una posible relación negativa en el tiempo entre el PIB per cápita y la tasa de crecimiento de contaminación ambiental medida a través de $\left(\mathrm{CO}_{2}\right.$ y $\mathrm{N}_{2} \mathrm{O}$ ), es decir, existe una convergencia a menores tasa de crecimiento de contaminación a medida que se incremental el nivel de ingreso (ver anexos).

Dado los argumentos anteriores, el objetivo de este trabajo es responder la siguiente pregunta: ¿Es posible compro- bar empíricamente la existencia de la curva medioambiental de Kuznets en las emisiones de dióxido de carbono y óxido nitroso durante el periodo 1990-2012? Los resultados anteriores permiten evidenciar la relación de factores que pueden generar impacto en el medio ambiente, los cuales están estrechamente relacionados con el crecimiento económico; sin embargo, estos no son suficientes. Por ello, la CMK llega como alternativa empírica para comprobar la hipótesis entre crecimiento económico y calidad ambiental, la cual busca no solamente contribuir al desarrollo del conocimiento, sino también establecer 
los impactos de la estructura económica en los bienes escasos (recursos naturales), que permitan generar información relevante para el diseño apropiado de estrategias en política pública en pro de un crecimiento económico eficiente y sostenible.
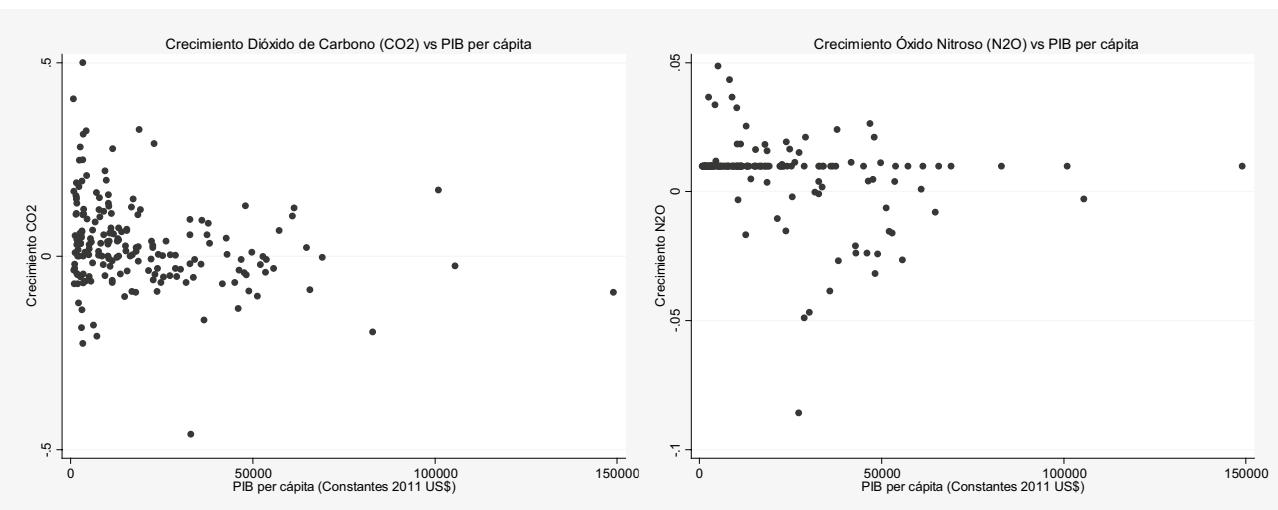

Figura 2. Crecimiento de dióxido de carbono $\left(\mathrm{CO}_{2}\right)$ y óxido nitroso $\left(\mathrm{N}_{2} \mathrm{O}\right)$ vs PIB per cápita para el año 2012

El artículo se organiza de la siguiente manera: esta introducción como primera sección; posteriormente en la sección de teoría y literatura se revisa la teoría económica utilizada, como también los antecedentes en el mundo, América Latina y Colombia; la sección de metodología y daros presenta los datos con las variables utilizadas y describe la metodología para la estimación de la CMK; en la sección cinco se muestran y analizan los resultados obtenidos y en la última sección se indican las conclusiones.

\section{TEORÍA Y LITERATURA}

La hipótesis de la curva de Kuznets presentada por Simon Kuznets (1955) en su trabajo titulado Economic Growth and Income Inequality, establece la relación del crecimiento económico y la desigualdad en el ingreso. El autor encuentra que existe una relación en forma de $U$ invertida ${ }^{1}$ a largo plazo entre el crecimiento económico y la distribución del ingreso. Para ello, utiliza el razonamiento deductivo2 que permita analizar los cambios en la distribución del ingreso, dado el proceso de crecimiento económico en los países desarrollados y subdesarrollados a fina-

$\overline{1}$ Se le conoce así a la relación entre variables que tienen tendencia estrictamente cóncava, es decir, para países con bajos ingresos existen elevados niveles de contaminación; sin embargo, al incrementarse el ingreso, los datos muestran una disminución en los indicadores de contaminación.

2 La falta de un modelo teórico formal razonable lleva a Kuznets a establecer un razonamiento basado en evidencia empírica para construir fundamentos que permitan analizar este posible fenómeno económico. 
les del siglo XIX y mediados del siglo $\mathrm{XX}$. Su estudio muestra que esta relación se puede explicar principalmente por tres factores: estructura poblacional, cambio en los sectores productivos y concentración del ahorro.

Estructura poblacional. Los cambios en la estructura demográfica en los países desarrollados han llevado a concentrar su población en la zona urbana, lo cual conduce a la población a emplearse en actividades que generen mejores ingresos y por ende un mayor crecimiento económico, que permita generar una movilidad en la distribución del ingreso a través del incremento en la participación del ingreso para los deciles más bajos.

Cambio en los sectores productivos. Establece que los cambios de la estructura productiva en favor del sector industrial con respecto al sector agrícola han llevado a incrementar el ingreso per cápita en toda la distribución del ingreso, principalmente para los deciles con menores ingreso. Este factor compensa el incremento en la desigualdad provocado por el aumento de mano de obra "inmigrante" asociado al sector agrícola.

Concentración del ahorro. El incremento del sector industrial a través de nuevas industrias, acompañado de un cambio tecnológico, genera un incremento en la productividad, lo cual conlleva un mejoramiento en la estructura del ingreso (laboral y excedente de ganancias), aumentando la concentración del ahorro, acumulación de factores productivos y por ende el crecimiento económico.

\section{Antecedentes}

El trabajo pionero que estudia la relación empírica entre el crecimiento económico y la contaminación ambiental fue el desarrollado por Grossman y Krueger (1991), el cual tiene como objetivo estimar los impactos ambientales que pueden traer los acuerdos de libre comercio en Estados Unidos. Los autores construyen una base de datos con dos fuentes de información para 42 países durante los años 1977, 1982 y 1988; la primera contiene los indicadores de contaminación (dióxido de sulfuro - $\mathrm{SO}_{2}$, partículas suspendidas en el aire y humo) reportados por el Sistema Global de Monitoreo Ambiental y la segunda incluye los datos del PIB per cápita recopilados por Summers y Heston (1991) como variable que mide el crecimiento económico. A partir de un modelo de datos panel, encuentran para dos indicadores de contaminación (dióxido de sulfuro - $\mathrm{SO}_{2} \mathrm{y}$ humo) que países con bajos niveles de actividad económica (PIB per cápita) tienen elevadas concentraciones de contaminación; sin embargo, al incrementar el PIB per cápita disminuye la contaminación 3 . Según ellos, países con ingresos elevados presentan un cambio a tecnologías que reduzcan la contami-

3 Con un punto de inflexión para países con un PIB per cápita entre 4000 y 5000 dólares. 
nación de los procesos productivos, así como fuertes regulaciones ambientales en la producción.

Existen diversas investigaciones empíricas que abordaron esta problemática. Shafik y Bandyopadhya (1992) exploran la relación entre el crecimiento económico 4 y la calidad ambientals de 149 países durante el periodo 1960-1990. Ellos, a través de un modelo de datos panel, encuentran evidencia de diferentes relaciones entre el crecimiento económico y los indicadores ambientales. Los indicadores que miden la contaminación del aire (partículas suspendidas en el aire y dióxido de sulfuro) y deforestación (cambio forestal y tasa de deforestación) muestran una relación de $\mathrm{U}$ invertida con relación al crecimiento económico, ya que países con elevados niveles de ingreso per cápita e inversión generan cambios en su transformación productiva a través de tecnología y medidas ambientales estrictas que permitan proteger los recursos naturales. Por su parte, los indicadores de contaminación del agua (agua limpia y disolución de oxígeno en los ríos) tienen un efecto negativo con respecto al crecimiento económico, los autores establecen que pueden deberse a externalidades en el costo de su tratamiento.
Grossman y Krueger (1995) elaboran un estudio estructural que permite evaluar la relación empírica entre el crecimiento económico y la contaminación ambiental. Para ello, utilizan el PIB per cápita como variable proxy del crecimiento económico y cuatro tipos de indicadores de contaminación ambiental6. Un primer grupo que explica la contaminación en el aire, conformado por el dióxido de sulfuro $\left(\mathrm{SO}_{2}\right)$, humo y partículas pesadas en el aire. El segundo grupo examina la calidad del agua en los ríos a través de la medición de los niveles de oxígeno, utilizando como indicadores los niveles de disolución de oxígeno, demanda biológica de oxígeno $(D B O)$, demanda química de oxígeno $(D Q O)$ y los niveles de nitrato. El tercer grupo integra los indicadores de coliforme fecal y coliforme total, para evaluar la contaminación patogénica generada por la falta de tratamiento de aguas residuales. Por último, el cuarto grupo representado por el plomo, cadmio, arsenio, mercurio y níquel, mide la concentración de partículas metálicas en el agua.

Los autores proponen un modelo de datos panel como instrumento metodológico que permita evaluar la relación entre el crecimiento económico y la contaminación ambiental. Los resultados obtenidos muestran que existe una cierta relación en forma de $\mathrm{U}$ invertida

\footnotetext{
$4 \quad$ Utilizan como variable de crecimiento el PIB per cápita.

5 Este estudio tiene como aporte el uso de indicadores ambientales que reflejan el impacto ambiental en toda su dimensión, los cuales son los siguientes: agua limpia, saneamiento básico, partículas suspendidas en el aire, dióxido de Sulfuro $\left(\mathrm{SO}_{2}\right)$, cambio forestal, tasa de deforestación y disolución de oxígeno en los ríos.

6 Los autores elaboraron una base de datos conformada por información del Banco Mundial y del Sistema Global de Monitoreo Ambiental.
} 
entre el crecimiento económico y la contaminación ambiental; encuentran que países con un PIB per cápita de 8000 y 10000 dólares tienen menores niveles de contaminación con respecto a países con menores ingresos. Ellos establecen que si bien existe una relación, esta no se genera de forma causal (determinística); sin embargo, empíricamente la relación entre el crecimiento y la contaminación no se puede rechazar.

Dado esto, los autores plantean una serie de hipótesis que buscan contribuir a explicar esta relación. En primer lugar, la contaminación conduce ex post a que los países con mayor desarrollo generen como respuesta una estricta regulación ambiental (política pública). Segundo, puede existir un cambio en las preferencias de consumo en los agentes por bienes no intensivos en contaminación. Finalmente, en tercer lugar, desde el punto de vista de la oferta, los países desarrollados con gran inversión en tecnología cambian su estructura productiva y procesos de producción a bienes menos intensivos en contaminación.

Por su parte, Selden y Song (1994) estudian la relación entre el crecimiento económico y la contaminación ambiental, específicamente para indicadores de calidad de aire?, utilizando un modelo de datos y como base de datos los reportes emitidos por el Sistema Global de Monitoreo Ambiental. Los resultados sugieren que existe una posible relación en forma de U invertida entre el PIB per cápita y todos los indicadores de contaminación del aire. Sin embargo, los autores establecen que si bien las emisiones se reducen, a largo plazo pueden incrementarse debido al aumento poblacional y, por ende, el uso de los recursos naturales.

Según Correa (2004), autores como Arrow et al. (1995), Stern et al. (1996), Ekins (1997) y Rothman (1998) establecen que la relación en forma de $\mathrm{U}$ invertida entre el crecimiento económico y la contaminación ambiente se puede explicar por el cambio en la estructura productiva hacia una producción limpia, el cual a su vez está acompañado por un cambio en las preferencias de los consumidores que lleva a que empresas migren a economías menos desarrolladas. Sin embargo, la evidencia empírica es insuficiente debido a la dificultad de obtener datos que relacionen los indicadores de contaminación ambiental con el comercio.

Así mismo, Andreoni y Levinson (1998) plantean que algunos indicadores de contaminación presentan una relación en forma de $\mathrm{U}$ invertida con respecto al ingreso per cápita; a través de un modelo estático encuentran que esta relación se debe principalmente a los rendimientos crecientes que genera el progreso tecnológico en la producción de bienes con menor uso de recursos, lo

7 Partículas suspendidas en el aire, dióxido de sulfuro (SO2), dióxido de nitrógeno (NO2) y monóxido de carbono (CO). 
que ocasiona una disminución de subproductos no deseables desde el punto de vista ambiental.

Bruyn et al. (1998) buscan comprobar la hipótesis de la CMK para Alemania Occidental, Holanda, Reino Unido y Estados Unidos durante el periodo 1960-19938. Para ello, establecen un modelo econométrico 9 basado en la intensidad de emisión de contaminantes, el cual refleja mejor el efecto de los cambios de estructura económica, tecnología, procesos productivos $\mathrm{y}$ políticas de regulación ambiental en los indicadores de contaminación (dióxido de carbono - $\mathrm{CO}_{2}$, óxido de nitrógeno $N O_{X}$ y dióxido de sulfuro - $S O_{2}$ ). Los resultados muestran que la intensidad en las emisiones de contaminación tiene una correlación positiva con el crecimiento económico; según ellos, la reducción en las emisiones puede explicarse por los cambios en la tecnología y por ende la estructura económica. Así mismo, al estimar el crecimiento sostenible ${ }^{10}$, encuentran que existe una compensación en la emisión de contaminación, entre el efecto positivo que puede traer el crecimiento y el efecto negativo del cambio en la tecnología y estructura económica11.

Por otro lado, un trabajo importante desde el punto de vista metodológico fue desarrollado por Stern y Common (2001), el cual busca comprobar empíricamente la existencia de la CMK empleando el azufre $(S)$ como indicador de contaminación ambiental; utiliza un conjunto de datos de 73 países durante el periodo de 1960-199012. Plantean que una debilidad en los modelos estructurales utilizados para estudiar la CMK consiste en la correcta especificación del modelo ${ }^{13}$, lo cual puede generar un sesgo en los estimadores; para solucionar parcialmente este problema elijen como variable explicada el azufre14, dividen la muestra en países desarrollados (incluidos en la OCDE) y en desarrollo (no incluidos en la OCDE); así mismo, utilizan el método de primeras diferencias para eliminar la heterogeneidad no observada en el tiempo. Ellos comprueban empíricamente la existencia de la CMK para los países desarrollados y no desarrollados; sin embargo, el punto

8 Construyen una base de datos con información de las siguientes autores e instituciones: Agencia Internacional de Energía, Banco Mundial, Organización para la Cooperación y el Desarrollo Económico (OCDE), Laboratorio Nacional de Oak Ridge, Agencia de Protección Ambiental, Baumol y Oates (1979), Oficina Federal de Estadística (Alemania) y la Oficina Central de Estadística (Holanda).

9 Difiere del modelo en forma reducida desarrollado por Grossman y Krueger (1995); aunque estima de manera directa la relación entre crecimiento económico y contaminación, no es claro por qué existe esa relación, es decir, es difícil interpretar los coeficientes.

10 Se entiende como la tasa de crecimiento económico que no conduce a un crecimiento en las emisiones de contaminantes.

11 Especialmente para la en ese entonces Alemania Occidental.

12 En las emisiones de azufre se utiliza información de la OCDE; con respeto al PIB per cápita se emplea la base de datos Penn World, desarrollada por la Universidad de California y el Centro de Crecimiento y Desarrollo de la Universidad de Groningen, para un total de 2263 observaciones.

13 Debido a que no existe un variable específica que explique el comportamiento de los indicadores de contaminación, es decir, puede generar un problema de sesgo por variables omitidas o simultaneidad, ya que la deforestación o emisión de contaminantes son determinantes de crecimiento económico.

14 Es una variable que puede tener menos problemas de simultaneidad, debido a tecnologías que hacen más fácil controlar su emisión. 
máximo de contaminación es menor para los países desarrollados. Con relación a los estimadores bajo el método de primeras diferencias, encuentran que un incremento en el ingreso (PIB per cápita) genera un aumento promedio en las emisiones de azufre de $43 \%$ y $55 \%$ para los países en desarrollo y desarrollados respectivamente.

Luzzati y Orsini (2009) estudian la relación entre el consumo de energía $\mathrm{y}$ el producto interno bruto (PIB) para 113 países durante el periodo 1971-2004; para ello, contribuyen metodológicamente a través de modelos semiparamétricos y el uso del consumo de energía como variable proxy de contaminación ambiental. Los autores encuentran que existe potencialmente evidencia de la CMK de energía, al estimar para tres subperiodos (1971-1981, 1982-1992, 1993-2004). No obstante, las estimaciones con respecto al tiempo muestran que no existe una evidencia sólida de la relación entre crecimiento económico y el consumo de energía 15.

Awaworyi et al. (2018) buscan estudiar el impacto de las emisiones de dióxido de carbono $\left(\mathrm{CO}_{2}\right)$ en el calentamiento global; para ello, pretenden probar la hipótesis de la CMK para 20 países integrantes de la OCDE durante el periodo 1870-2014. Con ayuda de un modelo de datos panel encuentran evidencia de la existencia de la CMK, específicamente para tres estimadores (MG, AMG y PMG) con puntos de inflexión implíci- tos entre un rango de 18955 a 89540 dólares. No obstante, al estimar por cada país, los autores hallan que solo cinco países tiene una relación de $\mathrm{U}$ invertida entre las emisiones de $\mathrm{CO}_{2}$ y el ingreso per cápita.

Finalmente, Shahbaz et al. (2020) analizan la relación entre el crecimiento económico y el consumo de energía de 30 provincias de China durante el periodo 1980-2018. Lo interesante es que los autores utilizan series de tiempo no paramétricas y datos panel como métodos de estimación. Ellos encuentran una relación en forma de $U$ invertida entre el consumo de energía y el ingreso per cápita en 20 provincias; no obstante, al aplicar el modelo no paramétrico se encuentra una correlación positiva promedio entre el crecimiento económico y consumo de energía.

Para América Latina existen trabajos que estudian la hipótesis de la CMK; sin embargo, se destacan por completo dos trabajos. El primero desarrollado por Saravia (2002), el cual estudia la hipótesis de la CMK para 17 países de Latinoamérica utilizando las emisiones de $\mathrm{CO}_{2}$ y $\mathrm{NO}_{2}$ como indicadores de calidad ambiental. Lo interesante de esta investigación es la estimación de los efectos que tiene la desigualdad en la distribución del ingreso y el desempeño institucional en el crecimiento económico, los cuales desaceleran el impacto positivo del crecimiento económico en la calidad ambiental,

15 Debido al crecimiento que tiene el consumo de energía en el tiempo. 
alejando el punto óptimo y por ende postergando la relación negativa entre el crecimiento económico y el deterioro de los recursos naturales.

Por su parte, Romero y De Jesús (2016) investigan la relación entre el crecimiento económico y el consumo de energía para 22 países de América Latina y el Caribe durante el periodo 1990-201116, utilizando como base de datos información del Banco Mundial. Los resultados evidencian que no existe una relación en forma de $U$ invertida entre el consumo de energía y el crecimiento económico (CMK de energía); por el contrario, muestran que en la región a medida que crecen sus economías también lo hace el consumo de energía. Es por ello que plantean políticas de eficiencia energética, que permitan adoptar cambios en la estructura productiva $\mathrm{y}$ promover medidas para incrementar el uso de energías no contaminantes.

En Colombia se destaca el estudio de Correa et al. (2005), el cual busca comprobar la hipótesis de la CMK para Colombia durante el periodo 19702000, utilizando $\mathrm{CO}_{2}, \mathrm{SO}_{2}$ y $\mathrm{DBO}$ como indicadores ambientales. Los autores solo encuentran un efecto positivo entre crecimiento económico y el deterioro ambiental, es decir, solo la fase creciente de CMK. Por otro lado, un incremento de la desigualdad reduce la contamina- ción (especialmente para el $\mathrm{SO}_{2}$ ); según ellos, la población con mejores ingresos tiene menores preferencias por el deterioro ambiental. Por último, concluyen que existen otros factores que determinan la contaminación ambiental, tales como políticas de regulación para el cuidado del medio ambiente y mejorar los derechos de propiedad de los recursos naturales, los cuales permitan generar crecimiento económico sin incrementar la degradación ambiental.

\section{METODOLOGÍA Y DATOS}

\section{Datos}

Se utiliza la base de datos del Banco Mundial (The World Bank, 2020), la cual contiene información de carácter global en diversas cronologías, asociada a los Indicadores del Desarrollo Mundial (IDM); información suministrada a través de fuentes oficiales de los diferentes países. Cabe resaltar que esta base de datos agrupa una gran cantidad de información (actualizada y precisa) con el fin de conocer el desarrollo mundial de los diferentes países y regiones. La base está constituida con información de siete regiones para un total de 264 países (incluyendo pequeños Estados). Para este trabajo se utiliza un total de 217 economías (se eliminan las regiones). Así mismo, esta base se divide en 21 indicadores (género, salud, educación, agricultura y desarrollo rural, cambio

16 Argentina, Barbados, Bolivia, Brasil, Chile, Colombia, Costa Rica, Cuba, Ecuador, El Salvador, Guatemala, Honduras, Jamaica, México, Nicaragua, Panamá, Paraguay, Perú, República Dominicana, Trinidad y Tobago, Uruguay y Venezuela. 
climático, ciencia y tecnología, desarrollo social, desarrollo urbano, eficacia de la ayuda, energía y minería, infraestructura, medio ambiente, pobreza y política económica y deuda). A continuación, se presenta la descripción de las variables utilizadas en esta investigación.

\section{Variables dependientes}

- Emisiones de dióxido de carbono $\mathrm{CO} 2$ (kt). Son las emisiones que provienen de la quema de combustibles fósiles y de la fabricación del cemento, incluyen el dióxido de carbono producido durante el consumo de combustibles sólidos, líquidos, gaseosos y de la quema de gas; se encuentra medida en kilotoneladas (kt) la cual es igual a 1000 toneladas.

- Emisiones de óxido nitroso $\mathrm{N}_{2} \mathrm{O}$. En miles de toneladas métricas, las emisiones de óxido nitroso $\mathrm{N}_{2} \mathrm{O}$ son las derivadas de la quema de biomasa agrícola, las actividades industriales, la gestión ganadera y procesos biológicos de suelos y océanos. Es considerado como un gas de efecto invernadero, que perturba el efecto radioactivo de la tierra y por ende de su temperatura. Estas emisiones son usadas como factor de relación entre una actividad y su impacto ambiental. La conversión a equivalentes con el dióxido de carbono $\mathrm{CO}_{2}$, que es la mayor fuente de gas de efecto invernadero, permite comparar otros gases y obtener su equivalencia, con el fin de establecer sus contribuciones individuales y totales al calentamiento global.

\section{Variables independientes}

Siguiendo la literatura, para analizar los efectos de las variables independientes utilizadas en los indicadores de contaminación, se consideran los siguientes enfoques:

a. Ingreso: un mayor ingreso cambia las preferencias de los consumidores a bienes con procesos y estándares de producción más limpia, debido a que se incrementa su disponibilidad a pagar (Correa et al., 2005).

b. Cambios en la estructura productiva: establece que una reducción en los niveles de contaminación se debe al cambio en los procesos productivos, este se representa por las variables de actividad económica. Variables: emisión de metano, uso de energía, valor agregado del sector servicios, industria e industria manufacturera.

c. Protección ambiental: una protección ambiental estricta, ya sea definida a través de legislación ambiental, política pública o el fortalecimiento de los recursos naturales, conlleva una restricción en los agentes económicos que no les permite generar deterioro ambiental. Variable: desgaste recursos naturales.

d. Población: un incremento en población está correlacionado positivamente con el ingreso, debido a que a 
medida que se aumenta la población, la economía incrementa sus agentes económicos (entre ellos, las empresas), lo cual genera un aumento en la producción y el consumo de bienes (Correa et al., 2005).

- PIB per cápita (a precios constantes 2011 USD). PIB per cápita basado en la paridad de poder adquisitivo (PPA), es un producto interno bruto convertido en dólares internacionales que utiliza tasas de paridad de poder adquisitivo; un dólar internacional tiene el mismo poder adquisitivo sobre el PIB que el dólar estadounidense en los Estados Unidos. El PIB a precios del comprador es la suma del valor añadido bruto de todos los productores residentes en el país más cualquier impuesto sobre los productos y menos las subvenciones no incluidas en el valor de los productos, se calcula sin hacer deducciones por depreciación de activos fabricados o por agotamiento y degradación de los recursos naturales.

- Uso de energía ( $k g$ equivalente de petróleo). Por PIB de 1000 dólares (PPA constante de 2010), se refiere al uso de energía primaria antes de la transformación a otros combustibles de uso final, que es igual a la producción tradicional más las importaciones y los cambios en las existencias, menos las exportaciones y los combustibles suministrados a los buques y aeronaves que se dedican al transporte internacional.
- Desgaste recursos naturales. Es el ahorro ajustado por agotamiento de los recursos naturales como porcentaje del ingreso nacional bruto (INB). Se entiende por agotamiento la suma del agotamiento forestal neto (es la renta de recursos unitarios por el exceso de cosecha de madera redonda sobre el crecimiento natural), más el agotamiento de la energía (relación entre el valor del stock de recursos energéticos y la vida útil restante de la reserva, cubre el carbón, el petróleo crudo y el gas natural) y el agotamiento mineral (es la relación entre el valor del stock de recursos minerales y la vida útil restante de la reserva, cubre estaño, oro, plomo, zinc, hierro, cobre, níquel, plata, bauxita y fosfato).

- Emisiones de metano $\mathrm{CH}_{4}(\mathrm{kt})$. Medidas en kilotoneladas ( $\mathrm{kt}$ ), la cual es igual a 1000 toneladas. Son las emisiones derivadas de actividades humanas como la agricultura, se producen debido a la descomposición o digestión de la materia orgánica y la producción industrial de metano. En esta investigación se realiza la equivalencia del metano con el $\mathrm{CO}_{2}$, con el fin de comparar las contribuciones efectivas de diferentes gases, si se tiene en cuenta que un kilogramo de metano es 21 veces más eficaz para atrapar el calor en la atmósfera terrestre que un kilogramo de dióxido de carbono en 100 años.

- Valor agregado del sector servicios (a precios constantes 2010 USD). Corresponde a las divisiones 50-99 
de la ISIC, que incluyen valor agregado en el comercio al por mayor $\mathrm{y}$ al por menor (incluidos hoteles y restaurantes), el transporte y los servicios gubernamentales, financieros, profesionales y personales, como educación, atención médica y servicios inmobiliarios; también se incluyen los cargos por servicios bancarios imputados, los derechos de importación y cualquier discrepancia estadística observada por los compiladores nacionales. El origen industrial del valor agregado está determinado por la Clasificación Industrial Estándar Internacional (IC), revisión 3 o 4.

- Valor agregado de la industria, incluida la construcción (a precios constantes 2010 USD). Corresponde a las divisiones 10-45 de la ISIC e incluye la fabricación (divisiones 15-37 del ISIC), comprende al valor agregado en minería, manufactura, construcción, electricidad, agua y gas; el origen de este valor está determinado por la Clasificación Industrial Estándar Internacional (ISIC), revisión 3.

- Valor agregado industria manufacturera (a precios constantes de 2010 USD). El término industrias manufactureras se refiere a las industrias pertenecientes a las divisiones 15 a 37 de la CIIU, donde el valor agregado es la producción neta de un sector después de sumar todos los productos y restar los insumos intermedios. Se calcula sin hacer deducciones por depreciación de bienes manufactura- dos o por agotamiento y degradación de recursos naturales, el origen del valor agregado de determina a partir de la CIIU, Revisión 3.

- Ahorro ajustado gasto en educación (\% del INB). El término gastos en educación se refiere a los gastos operativos corrientes en educación, que incluye sueldos y salarios, y excluye inversiones de capital en edificios y equipos.

- Población total. Se refiere a todos los residentes independientemente de su estatus legal o ciudadanía. Estas estimaciones de población se basan generalmente en censos nacionales de población y las estimaciones para los años anteriores y posteriores al censo son interpolaciones o extrapolaciones basadas en modelos demográficos (los errores pueden ser sustanciales debido a los límites en el transporte, las comunicaciones y otros recursos necesarios para llevar a cabo y analizar un censo completo, los cuales están sujetos a políticas, definiciones y conceptos internos de cada país).

\section{MODELO}

La presente investigación se basa en el nivel de investigación explicativo, en el cual los componentes se ajustan con la investigación no experimental (conocida como investigación "ex post facto"), debido a que busca cuantificar y comprobar empíricamente la existencia de la CMK, empleando variables no manipuladas que permitan ajustarse a 
este tipo de investigación. Siguiendo a Stern y Common (2001), se estima el logaritmo cuadrático de la CMK a través de un panel de datos, como se presenta a continuación:

$$
\ln \left(E_{i t}\right)=\alpha_{i}+v_{t}+\beta_{1} \ln (P I B)_{i t}+\beta_{2} \ln (P I B)_{i t}^{2}+\sum_{j=1}^{k} \beta_{j} \ln X_{j, i t}+u_{i t}
$$

Donde $E_{i t}$ indica la variable de contaminación ambiental $\left(\mathrm{CO}_{2}\right.$ y $\left.\mathrm{N}_{2} \mathrm{O}\right), \mathrm{PIB}$ es el producto interno bruto per cápita, $X_{j, i t}$ es la matriz de variables que intervienen en la contaminación ambiental (emisiones de metano, uso de energía, valor agregado del sector servicios, valor agregado de la industria, valor agregado de la manufactura, desgaste de los recursos naturales, educación y población), $u_{i t}$ es el término no observable (perturbación aleatoria), $\alpha_{i}$ es la heterogeneidad no observable para los países (unidades de sección cruzada) y $\mathrm{v}_{\mathrm{t}}$ incluye la heterogeneidad no observable en el tiempo. Así mismo, $\beta$ representa los estimadores y los subíndices $i, t$ indican observaciones para distintos individuos (países) y periodos de tiempo.

Para estimar la ecuación anterior se plantea utilizar tres alternativas. La primera, conocida como efectos fijos, establece que la heterogeneidad no observada $\left(\alpha_{i}\right)$ es una característica propia de los individuos (países); es por ello que el estimador ignora información de los cambios de las variables en el tiempo (Wooldridge, 2015), es decir, $\alpha_{i}$ está correlacionado con las variables independientes, lo que lleva a utilizar los promedios en el tiempo de las variables, para luego efectuar una regresión de corte transversal y así eliminar la heterogeneidad no observada. La segunda alternativa surge del resultado en el cual la heterogeneidad no observable de los individuos $\left(\alpha_{i}\right)$ no está correlacionada con las variables independientes, en otras palabras, $\alpha_{i}$ y $V_{t}$ son componentes de la perturbación aleatoria, esta se conoce como efectos aleatorios. No obstante, para establecer si la heterogeneidad no observada en los individuos (países) está correlacionada con las variables independientes y esto a su vez genera estimadores eficientes se utiliza la prueba de Hausman. Esta prueba compara los estimadores $\alpha_{i}$ y $V_{t}$ bajo los supuestos de efectos aleatorios, si la diferencia no es significativa, las estimaciones para efectos fijos y aleatorios son similares, es decir, no se rechaza. $\mathrm{Si}$ las estimaciones no son similares se debe a que las variables independientes están correlacionadas con $\alpha_{i}$ (no se cumple el supuesto fundamental de efectos aleatorios); por lo tanto, se utilizan las estimaciones de efectos fijos. Finalmente, la tercera alternativa consiste como en efectos fijos, en eliminar la heterogeneidad no observada a través de estimar el rezago entre las variables 
(método conocido como primera diferencias); sin embargo, esta alternativa tiene un costo en la pérdida de observaciones y a su vez se debe modificar la interpretación de sus resultados. Para calcular el PIB per cápita (ingreso) que genera los mayores niveles de contaminación (punto de inflexión) se utiliza la siguiente ecuación:

\section{RESULTADOS}

La Tabla 1 muestra los resultados de las tres alternativas de estimación para las emisiones de dióxido de carbono $\left(\mathrm{CO}_{2}\right)$; así mismo, se presenta al número de observaciones, $R$ cuadrado ${ }^{17}, R$ cuadrado within $18, R$ cuadrado between ${ }^{19}$, $R$ cuadrado overall 20 y la prueba de Hausman. Como se puede observar, las estimaciones comprueban que existe una relación de U-invertida entre las emisiones de dióxido de carbono $\left(\mathrm{CO}_{2}\right)$ y el PIB per cápita para primeras diferencias $\left(\beta_{1}=2.1522 ; \beta_{2}=-0.1107\right)$, efectos aleatorios $\left(\beta_{1}=3.5633 ; \beta_{2}=-0.1420\right) \mathrm{y}$ efectos fijos $\left(\beta_{1}=3.3755 ; \beta_{2}=-0.1305\right)$, siendo estadísticamente significativas al $1 \%$; no obstante, el efecto decreciente que tiene el ingreso (PIB per cápita) en la contaminación (emisiones de $\mathrm{CO}_{2}$ ) es muy bajo, es decir, un incremento en el ingreso disminuye muy poco las emisiones de $\mathrm{CO}_{2}$, lo cual conlleva puntos de inflexión implícitos demasiado elevados ${ }^{21}$ para la literatura existente ${ }^{22}$. En materia de producción de energía, un incremento del $1 \%$ en el uso de energía aumenta las emisiones dióxido de carbono en $0.827 \%, 0.895 \%$ y $0.918 \%$ para primeras diferencias, efectos aleatorios y efectos fijos respectivamente. Como era de esperarse, un aumento del $1 \%$ en el ahorro por evitar el agotamiento de los recursos naturales generados de la actividad productiva, reduce la contaminación ambiental por $\mathrm{CO}_{2}$ en $0.011 \%$ para efectos aleatorios y $0.0108 \%$ para efectos fijos. Con respecto a la actividad económica, los resultados muestran que las actividades de industria y servicios tienen una elasticidad promedio positiva en las emisiones de $\mathrm{CO}_{2}$ (excepto en primeras diferencias); no obstante, la actividad industrial manufacturera tiene un efecto negativo, esto se debe principalmente a que esta actividad económica se caracteriza por procesos productivos que reducen parcialmente la contaminación ambiental. El ahorro en el gasto de educación y la población incrementa las emisiones de dióxido de carbono. Finalmente, al aplicar la prueba de Hausman se establece que la

17 Se utiliza en primeras diferencias, debido a que en esta alternativa se emplea el Método de Mínimos Cuadrados Ordinarios (MCO).

18 Estima el efecto de la variabilidad en las variables independientes sobre las emisiones de contaminación ambiental (variable dependiente) en el tiempo.

19 Estima el efecto de la variabilidad en las variables independientes sobre las emisiones de contaminación ambiental (variable dependiente) entre individuos (países).

20 Estima el efecto total (within y between) de la variabilidad en las variables independientes sobre las emisiones de contaminación ambiental (variable dependiente).

21 16,662 dólares para primeras diferencias, 281,203 dólares para efectos aleatorios y 413,722 dólares para efectos fijos.

22 Stern y Common (2001) obtienen este tipo de estimaciones para países desarrollados y en desarrollo con puntos de inflexión de 101,666 y 908,178 dólares respectivamente, lo cual no contradice los resultados. 
heterogeneidad no observada entre países esta correlacionada con las variables independientes, esto implica que se debe utilizar el método de efectos fijos, cuya variabilidad en la contaminación está explicada principalmente por la variabilidad en las variables independientes entre países ( $R$ cuadrado between).

Tabla 1. Resultados de estimación para las emisiones de dióxido de carbono $\left(\mathrm{CO}_{2}\right)$

\begin{tabular}{|c|c|c|c|}
\hline Ln (dióxido de carbono) & Primeras diferencias & Efectos aleatorios & Efectos fijos \\
\hline \multirow{2}{*}{$\operatorname{Ln}(\mathrm{PIB})$} & $2.1522^{* * *}$ & $3.5633^{* * *}$ & $3.3755^{* * *}$ \\
\hline & $(0.4400)$ & $(0.1773)$ & $(0.1963)$ \\
\hline \multirow{2}{*}{ Ln (PIB) cuadrado } & $-0.1107^{* * *}$ & $-0.1420^{* * *}$ & $-0.1305^{* * *}$ \\
\hline & $(0.0234)$ & $(0.0081)$ & $(0.0087)$ \\
\hline \multirow{2}{*}{ Ln (Emisión metano) } & $0.0568^{* * *}$ & $0.1100^{* * *}$ & $0.1407^{* * *}$ \\
\hline & $(0.0176)$ & $(0.0195)$ & $(0.0208)$ \\
\hline \multirow{2}{*}{ Ln (Uso de energía) } & $0.8273^{* * *}$ & $0.8955^{* * *}$ & $0.9182^{* * *}$ \\
\hline & $(0.0410)$ & $(0.0267)$ & $(0.0301)$ \\
\hline \multirow{2}{*}{ Ln (PIB Servicios) } & $-0.1112^{*}$ & $0.1086^{* * *}$ & 0.0541 \\
\hline & $(0.0620)$ & $(0.0353)$ & $(0.0403)$ \\
\hline \multirow{2}{*}{ Ln (PIB Industria) } & $0.1043^{* *}$ & $0.2610^{* * *}$ & $0.2837^{* * *}$ \\
\hline & $(0.0520)$ & $(0.0342)$ & $(0.0380)$ \\
\hline \multirow{2}{*}{ Ln (PIB Manufacturera) } & -0.0446 & $-0.2305^{* * *}$ & $-0.2519^{* * *}$ \\
\hline & $(0.0344)$ & $(0.0198)$ & $(0.0202)$ \\
\hline \multirow{2}{*}{ Ln (Educación) } & 0.0031 & $0.1156^{* * *}$ & $0.1130 * * *$ \\
\hline & $(0.0182)$ & $(0.0133)$ & $(0.0132)$ \\
\hline \multirow{2}{*}{ Ln (Población) } & $0.7017^{* * *}$ & $0.7511^{* * *}$ & $0.9008^{* * *}$ \\
\hline & $(0.2191)$ & $(0.0599)$ & $(0.0765)$ \\
\hline \multirow{2}{*}{ Ln (Recursos naturales) } & 0.0019 & $-0.0111^{* * *}$ & $-0.0108^{* * *}$ \\
\hline & $(0.0033)$ & $(0.0031)$ & $(0.0031)$ \\
\hline $\mathrm{N}$ observaciones & 1975 & 2124 & 2124 \\
\hline \multirow{6}{*}{$\begin{array}{c}\text { R cuadrado } \\
\text { R cuadrado (within) } \\
\text { R cuadrado (between) } \\
\text { R cuadrado (overall) } \\
\text { Test Hausman } \\
\text { Prob (Chi2) }\end{array}$} & 0.208 & - & - \\
\hline & - & 0.776 & 0.778 \\
\hline & - & 0.931 & 0.918 \\
\hline & - & 0.935 & 0.915 \\
\hline & - & - & 268.29 \\
\hline & - & - & $(0.0000)$ \\
\hline
\end{tabular}

Nota: $* \mathrm{p}<0.1 ; * * \mathrm{p}<0.05 ; * * * \mathrm{p}<0.01$.

Fuente: Elaboración propia con base en información del Banco Mundial.

De igual manera, la Tabla 2 presenta las estimaciones para el óxido nitroso $\left(\mathrm{N}_{2} \mathrm{O}\right)$. Los resultados muestran que existe una relación de $\mathrm{U}$ invertida entre las emisiones de $\mathrm{N}_{2} \mathrm{O}$ y el PIB per cápita para efectos fijos $\left(\beta_{1}=3.3755 ; \beta_{2}=-0.1305\right)$, estadísticamente significativo al $1 \%$, con un punto de inflexión implícito de 20786 dólares. Según la prueba de Hausman, el método de efectos fijos es consistente, debido a la correlación que existe entre las variables independien- 
tes y la heterogeneidad no observada entre países; así mismo, en términos de variabilidad, se puede observar que en el método de efectos fijos el $86 \%$ de la variabilidad en las emisiones de $\mathrm{N}_{2} \mathrm{O}$ se explica por los cambios en las variables independientes entre países $(R$ between). Con relación a la eficiencia energética, los resultados establecen que un incremento en $1 \%$ en el uso de energía aumenta la contaminación por óxido nitroso en $0.167 \%$; en materia de protección de los recursos naturales, un aumento de $1 \%$ en el ahorro para evitar agotamiento de los recursos naturales, reduce las emisiones de $\mathrm{N}_{2} \mathrm{O}$ en $0.043 \%$. Las estimaciones para la actividad económica muestran que un aumento de la industria y los servicios 23 tiene un efecto negativo; todo lo contrario sucede con la industria manufacturera, la cual tiene un efecto positivo en las emisiones de óxido nitroso. Por último, el gasto en educación y la población tiene efectos contrarios, el primero incrementa la emisión de $\mathrm{N}_{2} \mathrm{O}$ y el segundo la reduce.

Tabla 2. Resultados de estimación para las emisiones de óxido nitroso $\left(\mathrm{N}_{2} \mathrm{O}\right)$

\begin{tabular}{|c|c|c|c|}
\hline $\begin{array}{l}\text { Ln (Dióxido de } \\
\text { Nitrógeno) }\end{array}$ & Primeras diferencias & Efectos aleatorios & Efectos fijos \\
\hline \multirow[t]{2}{*}{$\operatorname{Ln}(\mathrm{PIB})$} & -0.5774 & 0.2562 & $0.6860^{* * *}$ \\
\hline & $(0.4052)$ & $(0.1945)$ & $(0.2170)$ \\
\hline \multirow[t]{2}{*}{ Ln (PIB) cuadrado } & 0.0320 & $-0.0221^{* *}$ & $-0.0345^{* * *}$ \\
\hline & $(0.0215)$ & $(0.0090)$ & $(0.0096)$ \\
\hline \multirow[t]{2}{*}{ Ln (Emisión Metano) } & $1.1905^{* * *}$ & $1.0258^{* * *}$ & $1.0161^{* * *}$ \\
\hline & $(0.0162)$ & $(0.0215)$ & $(0.0230)$ \\
\hline \multirow[t]{2}{*}{ Ln (Uso de energía) } & -0.0312 & $0.0680^{* *}$ & $0.1671^{* * *}$ \\
\hline & $(0.0378)$ & $(0.0292)$ & $(0.0332)$ \\
\hline \multirow[t]{2}{*}{ Ln (PIB Servicios) } & 0.0169 & 0.0251 & -0.0159 \\
\hline & $(0.0571)$ & $(0.0387)$ & $(0.0446)$ \\
\hline \multirow[t]{2}{*}{ Ln (PIB Industria) } & $-0.1304^{* * *}$ & -0.0479 & -0.0538 \\
\hline & $(0.0479)$ & $(0.0375)$ & $(0.0420)$ \\
\hline \multirow[t]{2}{*}{ Ln (PIB Manufacturera) } & $0.1413^{* * *}$ & $0.0836^{* * *}$ & $0.0540^{* *}$ \\
\hline & $(0.0317)$ & $(0.0218)$ & $(0.0223)$ \\
\hline \multirow[t]{2}{*}{ Ln (Educación) } & $0.0414^{* *}$ & $0.0758^{\star \star \star}$ & $0.0644^{\star \star *}$ \\
\hline & $(0.0168)$ & $(0.0147)$ & $(0.0146)$ \\
\hline \multirow[t]{2}{*}{ Ln (Población) } & 0.2293 & $-0.1522^{\star \star}$ & $-0.2694^{* * *}$ \\
\hline & $(0.2018)$ & $(0.0653)$ & $(0.0846)$ \\
\hline \multirow[t]{2}{*}{ Ln (Recursos naturales) } & $-0.0054^{*}$ & $-0.0488^{* * *}$ & $-0.0431^{* * *}$ \\
\hline & $(0.0031)$ & $(0.0034)$ & $(0.0035)$ \\
\hline N observaciones & 1975 & 2124 & 2124 \\
\hline R cuadrado & 0.735 & - & - \\
\hline R cuadrado (within) & - & 0.564 & 0.564 \\
\hline R cuadrado (between) & - & 0.902 & 0.856 \\
\hline
\end{tabular}

$\overline{23}$ A pesar de no ser estadísticamente significativo. 
Continuación Tabla 2

\begin{tabular}{cccc} 
R cuadrado (overall) & - & 0.904 & 0.860 \\
Test Hausman & - & - & 961.11 \\
Prob (Chi2) & - & - & $(0.0000)$ \\
\hline
\end{tabular}

Nota: ${ }^{*} \mathrm{p}<0.1 ;{ }^{* *} \mathrm{p}<0.05 ; * * * \mathrm{p}<0.01$.

Fuente: elaboración propia con base en información del Banco Mundial.

\section{CONCLUSIONES}

Es importante reconocer que esta investigación busca construir una estructura formal a través de una base inestable de causalidad, donde la formalidad juega un papel fundamental en el análisis económico; sin embargo, una visión general lleva a que el campo del conocimiento abarque más allá de ella (Kuznets, 1955). Por ello, este trabajo busca contribuir con el enriquecimiento del análisis entre el crecimiento económico y los efectos en el medioambiente, los cuales son el resultado de incentivos que hacen al análisis económico un complemento.

Los resultados muestran una relación en forma de $\mathrm{U}$ invertida entre la contaminación ambiental $\left(\mathrm{CO}_{2}\right.$ y $\left.\mathrm{N}_{2} \mathrm{O}\right)$ y el crecimiento económico (se comprueba la CMK); sin embargo, se encuentra puntos de inflexión implícitos elevados con respecto a los niveles de ingresos para el conjunto de países (principalmente para las emisiones de dióxido de carbono), es decir, el efecto entre contaminación y el nivel de ingreso presenta rendimientos decrecientes muy bajos. En materia de uso de energía se encuentra una elasticidad promedio positiva con respecto a los indicadores de contaminación $\left(0.918 \%\right.$ y $0.167 \%$ para $\mathrm{CO} 2$ y $\mathrm{N}_{2} \mathrm{O}$ respectivamente); igualmente sucede con la variable proxy de protección ambiental (ahorro por evitar el agotamiento de los recursos naturales), la cual reduce las emisiones de dióxido de carbono y óxido nitroso. Con respecto a la actividad económica, los resultados muestran diferentes efectos para ambos indicadores de contaminación; un incremento de $1 \%$ en el PIB del sector industrial incrementa las emisiones de $\mathrm{CO}_{2}$ y reduce las emisiones de $\mathrm{N}_{2} \mathrm{O}$.

La prueba de Hausman muestra que el método de efectos fijos genera estimadores consistentes, ya que la heterogeneidad no observada entre países está correlacionada con las variables independientes; sin embargo, es posible que existan diferentes efectos para diferentes muestras; por ello, se recomienda realizar diversas estimaciones para varias regiones, que permitan captar mejor el efecto del ingreso en la contaminación ambiental.

Por lo tanto, se puede concluir que, si bien el crecimiento económico genera un efecto creciente y decreciente en el deterioro ambiental, para ello, también 
es necesario un cambio en la estructura productiva que permita el uso de factores productivos hacia una producción sostenible. Así mismo, el crecimiento económico debe ir de la mano de una política de protección ambiental estricta. Como anotan Correa et al. (2005), se necesitan regulaciones a fin de cuidar el medio ambiente, por ejemplo, mejorar y hacer efectivos los derechos de propiedad de los recursos ambientales que no han sido asignados, lo cual puede evitar la degradación ambiental. De igual manera, es necesario evaluar y reafianzar el trabajo conjunto de los diferentes gobiernos, pues si bien el Acuerdo de París es un gran avance, no es suficiente para generar una política ambiental sostenible. Por su parte, en la educación, que es uno de los pilares de la formación de individuos sociales, se deben enfocar los esfuerzos en generar programas educativos que formen ciudadanos con conciencia ambiental, ya que, como se analizó anteriormente, los gastos operativos en educación no son suficientes.

Por último, pero no menos importante, la solución a esta problemática planteada va más allá del uso de bases de datos representativas o modelos cada vez más sofisticados, pues la idea es establecer una posible estructura que pueda determinar los efectos del crecimiento económico en la contaminación ambiental tanto a corto como a largo plazo; no obstante, inferir este efecto es complejo debido a las diversas decisiones de los agentes (países) y aún más si los resultados pueden tener un problema de endogeneidad (doble causalidad), ya que el crecimiento económico puede ser la causa del uso intensivo de los recursos naturales. Sin embargo, contribuir a la revisión y evolución de este tema en el tiempo, permite hacer un seguimiento $y$, sobre todo, estructurar lineamientos de políticas que se ajusten en cierta medida a la evidencia empírica.

\section{AGRADECIMIENTOS}

A la Universidad Surcolombiana, por la disponibilidad de tiempo por parte de los autores.

A los pares evaluadores de este artículo, sus comentarios enriquecieron el artículo.

\section{CONTRIBUCIÓN DE LOS AUTORES}

El trabajo es el resultado del trabajo en equipo de los autores.

\section{DECLARACIÓN DE CONFLICTOS DE INTERESES}

Los autores declaran que no existe conflicto de intereses.

\section{FINANCIAMIENTO}

Esta investigación se realizó con recursos propios. 


\section{REFERENCIAS}

[1] Andreoni, J., \& Levinson, A. (1998). The Simple Analytics of the Enviromental Kuznets Curve. NBER Working Paper Series, 15. National Bareau of Economic Research. https://doi.org/10.3386/w6739

[2] Arrow, S. et al. (1995). The Environmental Kuznets Curve in the OECD: 18702014 . Energy Economics.

[3] Awaworyi, S. et al. (2018). The Environmental Kuznets Curve in the OECD: 18702014. Energy Economics.

[4] Bruyn, S., Van den Bergh, J., \& Opschoor, J. (1998). Economic Growth and Emissions: Reconsidering the Empirical Basis of Environmental Kuznets Curves. Ecological Economics, 25, 161-175. HYPERLINK https://doi.org/10.1016/ S0921-8009(97)00178-X

[5] Correa, F. (2004). Crecimiento económico y medio ambiente: una revisón análitica de la hipótesis de la Curva Ambiental de Kuznets. Semestre Económico, 8(15).

[6] Correa, F., Vasco, A., \& Montoya, C. (2005). La Curva Medioambiental de Kuznets: evidencia empírica para Colombia. Semestre Económico, 8(15).

[7] Ekins, P. (1997). The Kuznets Curve for the Environment and Economic Growth: Examining the Evidence. Environment and Planning, 29, 805-830. HYPERLINK https://doi.org/10.1068/a290805

[8] Grossman, G. \& Krueger, A. (1991). Environmental Impact of a North American Free Trade Agreement. National Bareu of Economic Research. HYPERLINK https://doi.org/10.3386/w3914

[9] Grossman, G., \& Krueger, A. (1995). Economic Growth and the Environment. The Quarterly Journal of Economics, 110(2), 353-377. https://doi.org/10.2307/2118443

[10] Kuznets, S. (1955). Economic Growth and Income Inequality. American Economic Review, 45(1), 1-28.

[11] Luzzati, T., \& Orsini, M. (2009). Investigating the Energy-Environmental Kuznets Curve. https://doi.org/10.1016/j.energy.2008.07.006

[12] Romero, P., \& De Jesús, J. (2016). Economic Growth and Energy Consumption: The Energy-Environmental Kuznets Curve for Latin America and the Caribbean. Renewable and Sustainable Energy Reviews, 60, 1343-1350. HYPERLINK https://doi.org/10.1016/j.rser.2016.03.029 
[13] Rothman, D. (1998). Environmental Kuzntes Curve. Real Progress or Passing The Buck? A Case for Consumption. Based Approaches. Ecological Economics, 25(2), 177-194. HYPERLINK https://doi.org/10.1016/S0921-8009(97)00179-1

[14] Saravia, A. (2002). La curva medioambiental de Kuznets para América Latina y el Caribe. Documentos de Reflexión Académica, 23.

[15] Selden, T., \& Song, D. (1994). Environmental Quality and Development: Is There a Kuznets Curve for Air Pollution Emissions? Journal of Environmental Economics and Management, (27), 147-162. https://doi.org/10.1006/jeem.1994.1031

[16] Shafik, N., \& Bandyopadhya, S. (1992). Economic Growth and Environmental Quality Time-Series and Cross-Country Evidenc. World Bank, Working Papers, WPS 094.

[17] Shahbaz, M. et al. (2020). A Nonparametric Analysis of Energy Environmental Kuznets Curve in Chinese Provinces. Energy Economics. HYPERLINK "https:// doi.org/10.1016/j.eneco.2020.104814"

[18] Stern, D., Common, S., \& Barbier, E. (1996). Economic Growth and Environmental Degradation: A Critique of the Environmental Kuznets Curve. World Development, 24, 1151-1160. HYPERLINK https://doi.org/10.1016/0305-750X(96)00032-0

[19] Stern, D., \& Common, M. (2001). Is there An Environmental Kuznets Curve For Sulphur? Journal of Environmental Economics and Management, (41), 162-178. https://doi.org/10.1006/jeem.2000.1132

[20] The World Bank. (2020, 18 de mayo). Banco Mundial. htpp://www.datos.bancomundial.org

[21] Wooldridge, J. (2015). Introducción a la econometría. (Vol. 5). Cenage Learning. 


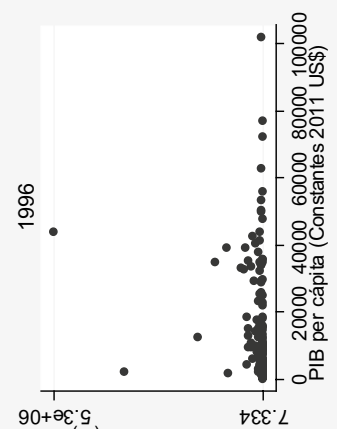

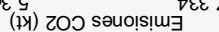

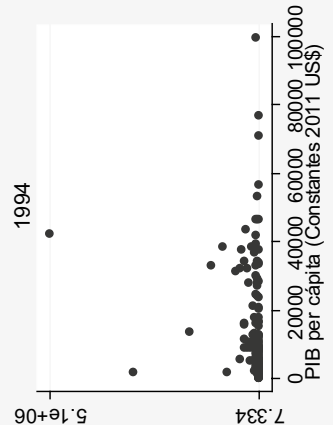

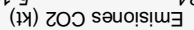

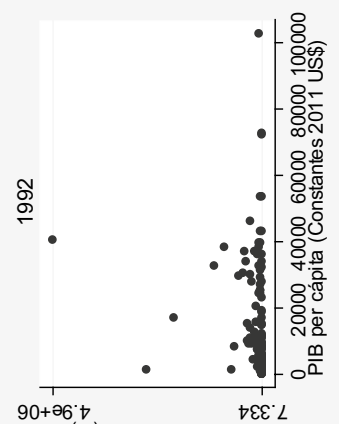

(ły) zOว səuo!s!m $\exists \varepsilon$

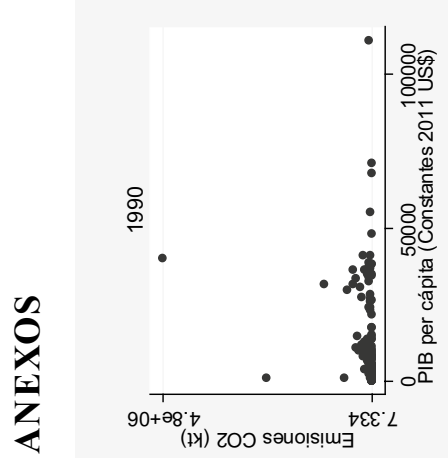

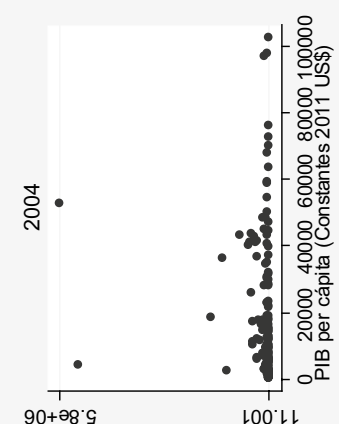

(1צ) zOว sәuo!s!̣u

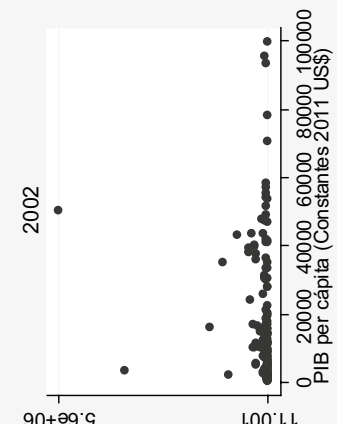

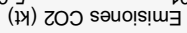

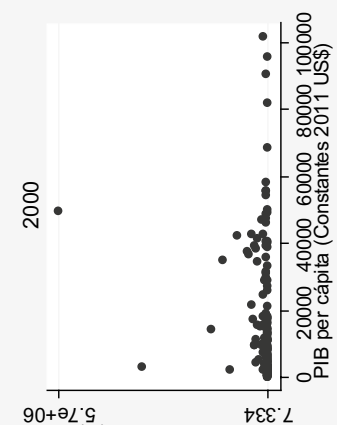

(14) zO० səuo!s!uヨ

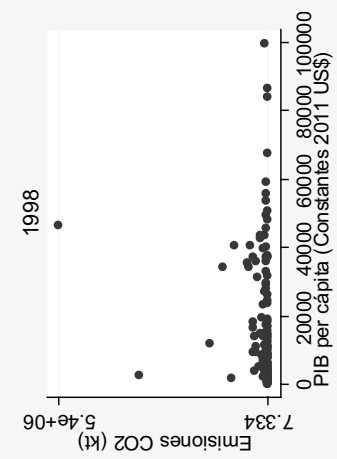

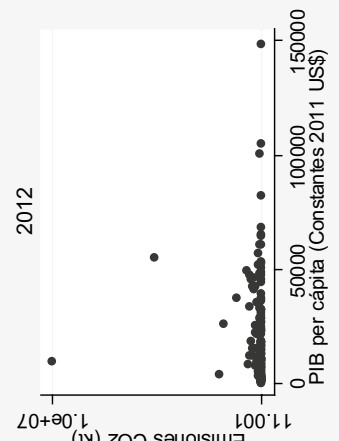

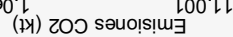

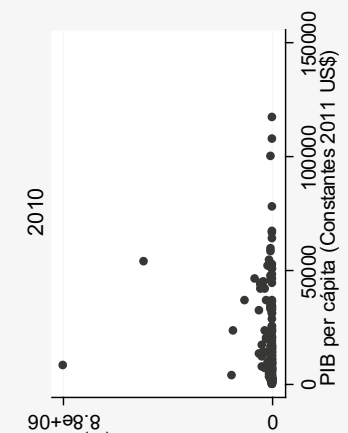

(ұ) 乙Оว sәuo!s!uヨ

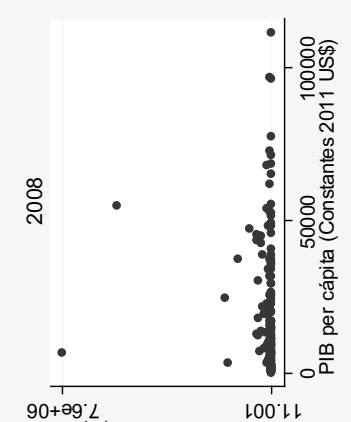

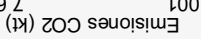

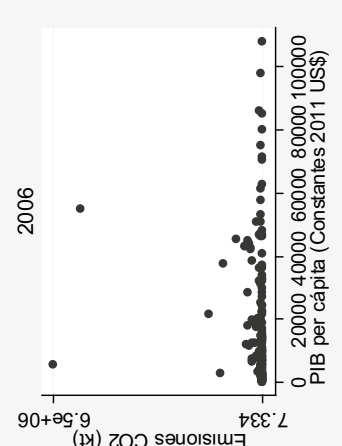

(ły) ZOว səuo!s!̣u 

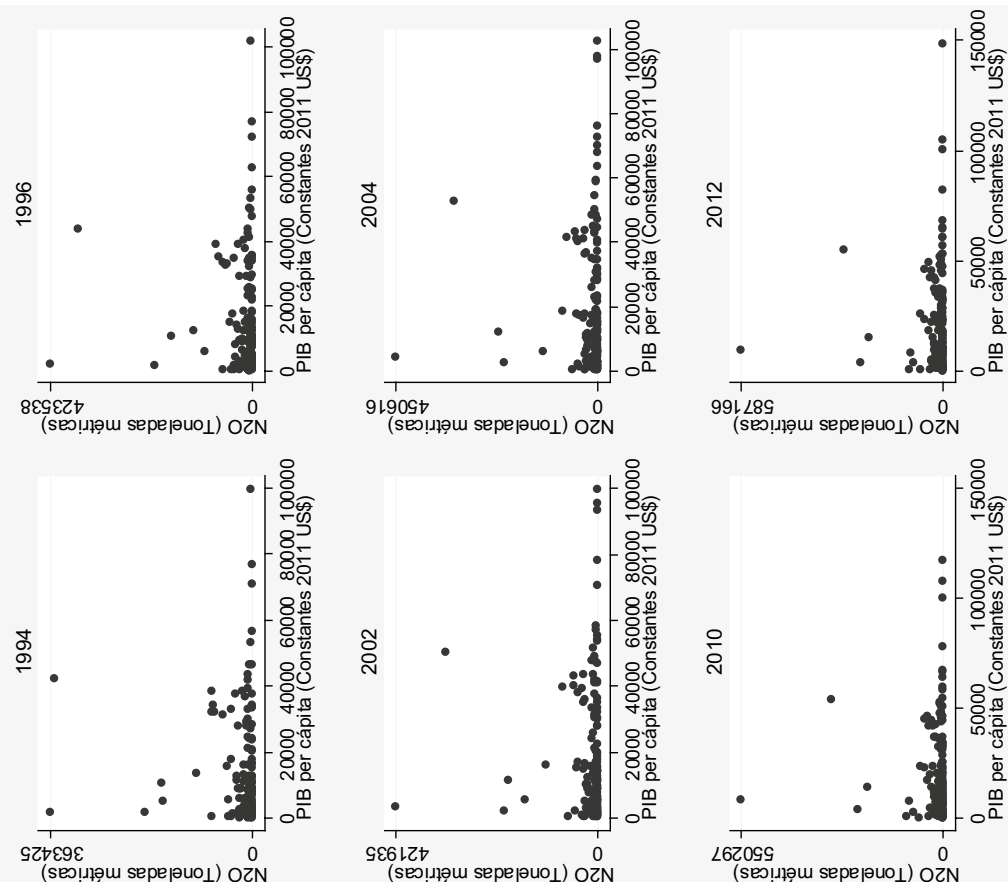

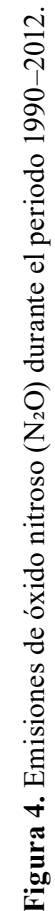
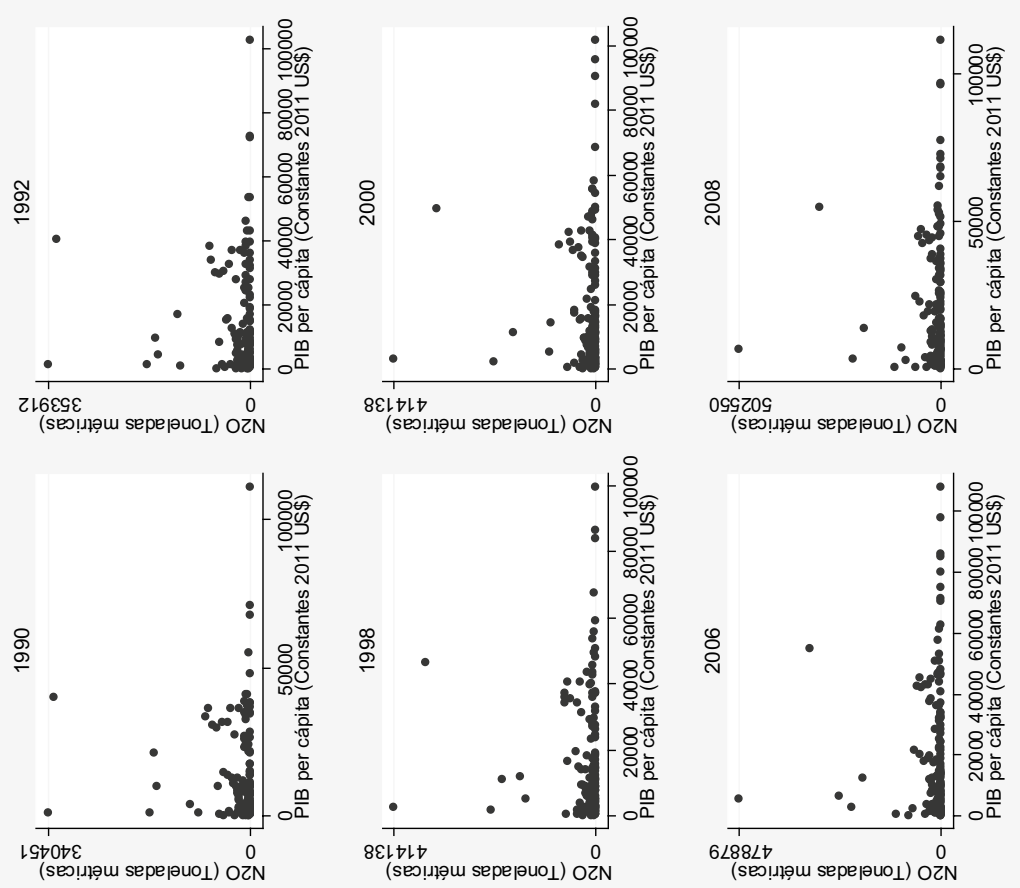